\title{
Structure-Unbinding Kinetics Relationship of p38a MAPK Inhibitors
}

\section{Author List}

Xiaoxia Ge ${ }^{*} \dagger, \S$, Hepan Tan ${ }^{\ddagger}$, and Lei Xie ${ }^{*, \S, " ~}$

\section{Affiliations}

${ }^{\dagger}$ School of Medicine, Nankai University, Tianjin, 300071, China

†jixun Computational Solutions, LLC., White Plains, NY, 10601, USA

${ }^{\S}$ Department of Computer Science, Hunter College, The City University of New York, New York, NY 10065, USA

"The Graduate Center, The City University of New York, New York, NY 10016, USA

\section{Corresponding Authors}

*X.G.: e-mail, xge@ opencadd.org;

*L.X.: e-mail, lei.xie@hunter.cuny.edu 


\begin{abstract}
Rational Drug Design still faces a major hurdle for the prediction of drug efficacy in vivo solely based on its binding affinity for the target in vitro. The traditional perspective has proven to be inadequate as it lacks the consideration of essential aspects such as pharmacokinetics and binding kinetics in determining drug efficacy and toxicity. Residence time, the average lifetime of drug-target complex, has gained broader recognition as a better predictor for lead optimization. Long residence time could contribute to sustained pharmacological effect and may mitigate offtarget toxicity as well. To unravel the underlining mechanism for variation of residence time and determine the ligand features governing the unbinding kinetics, unbinding kinetics of two distinct type II inhibitors of p38 $\alpha$ MAP kinase were investigated and compared by molecular dynamics and metadynamics simulation approaches. Free energy landscape of key motions associated with unbinding was constructed for both inhibitors. Multiple unbinding pathways and rebinding were revealed during the drug-target dissociation process of faster unbinder Lig3 and slower unbinder Lig8 respectively, suggesting a novel mechanism of unbinding kinetics. This comparative study implies that hydrophobic and hydrogen-bonding interactions in the R1 group of ligands are crucial for slow unbinding. Such kind of structure-kinetics relationship approaches could also be applied to predict unbinding pathways and kinetics of many other small molecules, and facilitate the design of efficient kinase inhibitors.
\end{abstract}

Keywords: drug efficacy, drug residence time, unbinding kinetics, unbinding pathway, MD simulation, metadynamics, p38 $\alpha$ MAP kinase inhibitor, molecular recognition 


\section{Introduction}

Molecular recognition is central to most biological processes. Among many types of molecular recognitions, drug-target recognition has been studied extensively due to their importance in understanding the mechanism of drug action and consequently improving the drug development process. ${ }^{1}$ Equilibrium binding metrics obtained from in vitro assays include the halfmaximal inhibitory concentration (IC50), the equilibrium dissociation constant $\left(K_{\mathrm{d}}\right)$ and the inhibition constant $\left(K_{\mathrm{i}}\right)$, etc. However, in vivo pharmacology often deals with open, nonequilibrium conditions, in which cases equilibrium assumption of drug-target interactions become invalid. Therefore, lead optimization solely based on improving the drug-target binding affinity does not necessarily improve drug efficacy in vivo. Residence time, defined as the lifetime of the drug-target complex, is a more relevant in vivo metric. ${ }^{2}$ Because long residence time leads to sustained pharmacology, drugs can deliver desirable efficacy at low doses. Lower doses may mitigate off-target toxicity and therefore reduce adverse drug effect (side effect). A static view of the drug-target interaction is inadequate to explain the impact of conformational dynamics on the binding and unbinding processes. For high-affinity interactions, both binding and unbinding processes involve conformational changes of the target. ${ }^{3}$ Conformational change associated with ligand binding/unbinding pathway is an important aspect in understanding the kinetic process.

In the past decade, a number of examples were reported on successful design of new drugs aiming to improve the residence time such as Triazole-Based InhA Inhibitors, ${ }^{4} \mathrm{CDK} 8$ inhibitors, ${ }^{5}$ and series of reversible covalent inhibitors. ${ }^{6-7}$ The prediction of kinetic rates based on residue coarse-grained normal mode analysis has been developed and tested successfully for HIV protease inhibitors. $^{8}$ 
Molecular dynamics (MD) simulation provides a view of the atomic motion over time. It has been a powerful tool for studying biological systems and provided many insights in different areas of research. ${ }^{9-10}$ Drug residence time spans from nanoseconds to hours in time scale. MD simulation results are meaningful only if the trajectories are long enough for the system to visit all the energetically relevant configurations. Practically, some biologically important configurations are often separated by high free-energy barriers; or the system diffuses extremely slowly rendering simulations to be beyond the limit of current computer resources. To tackle these issues, enhanced sampling techniques have been developed in the past few decades including replica exchange, ${ }^{11}$ accelerated MD, ${ }^{12}$ umbrella sampling, ${ }^{13}$ steered MD,${ }^{14}$ and metadynamics. ${ }^{15-17}$ These methods facilitate the crossing of free energy barrier via biasing system potential energy or biasing a few selected degrees of freedom, i.e., collective variables (CVs). Multiple review articles appeared recently on computational approaches for drug-binding/unbinding kinetics. ${ }^{18-19}$ Metadynamics reconstructs the free energy surface (FES) as a function of the selected CVs using a historydependent bias potential. Because of its broad applications in thermodynamic and kinetic problems, ${ }^{20-21}$ metadynamics approach has gained increasing attentions.

Protein kinases participate in a number of cellular signaling pathways by catalyzing the phosphorylation of specific substrates, whereby serving as an important family of drug targets. The catalytic activity of a kinase is regulated by its conformation plasticity, i.e., it switches between active and inactive forms. ${ }^{22} \mathrm{P} 38$ mitogen-activated protein kinases (MAPK) are a class of signal transduction mediators that play pivotal roles in inflammation, cell cycle, apoptosis, cell growth, cell differentiation, senescence and tumorigenesis depending on cell types. ${ }^{23}$ Among four MAPK isoforms $\mathrm{p} 38 \alpha, \beta, \gamma$, and $\delta$, the major one $\mathrm{p} 38 \alpha$ has been identified as drug targets for various inflammatory diseases, cancers, and cardiovascular diseases. ${ }^{24-26}$ Consequently, p38 $\alpha$ 
MAPK has been one of the mostly studied kinases with high-resolution crystal structures available for both the native and type II inhibitor-bound states. ${ }^{27}$ The crystal structure consists a common ATP-binding pocket and a novel allosteric binding site for a class of highly potent and selective diaryl urea inhibitors against human p38 $\alpha$ MAPK (Figure 1). The formation of this binding site requires a large conformational change of conserved Asp168-Phe169-Gly170 (DFG) motif not observed previously for any of Ser/Thr kinases. Solution studies demonstrate that this class of compounds has slow binding kinetics, consistent with the requirement for conformational change.

One of the most potent compounds in this series, 1-(5-tert-butyl-2-p-tolyl-2H-pyrazol-3-yl)-3-[4(2-morpholin-4-yl-ethoxy) naphthalen-1-yl]- urea (BIRB796), has picomolar affinity with human p38 $\alpha$ MAPK and low nanomolar inhibitory activity in cell culture. ${ }^{27-28}$
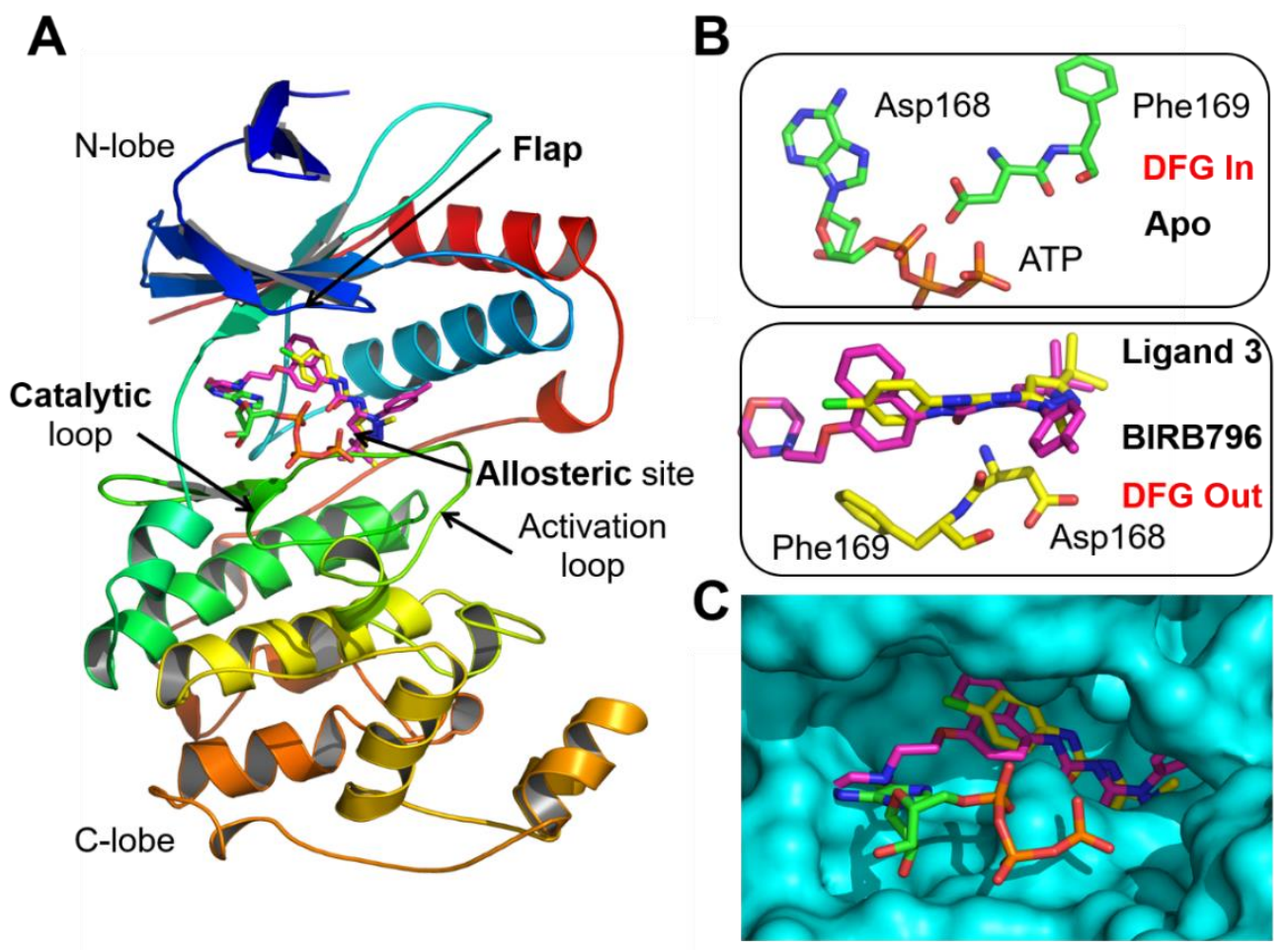

Figure 1. Binding site of the type II inhibitors on p38 $\alpha$ MAP kinase. A) A schematic view of the structure of p38 $\alpha$ in complex with BIRB796 and Lig3. B) DFG conformation change upon ligand binding. C) Superposition of p38a ligand-binding pocket. The molecular representations were generated with PyMOL ${ }^{29}$ based on PDB structures $1 \mathrm{P} 38,{ }^{30} 1 \mathrm{~K} 1 \mathrm{~V}$ and $1 \mathrm{KV} 2 .{ }^{27}$ 
Quite a few computational studies were carried out in the past few years on this important system, including binding free energy calculations of imatinib, sorafenib, BI-1 and BIRB-796 to $\mathrm{p} 38 \alpha^{31}$ These studies reveal that the driving force of binding was van der Waals interaction, typically hydrogen bonding. ${ }^{31}$ Sun et al. ${ }^{32}$ found that type II inhibitors unbind through two pathways in opposite directions, i.e., allosteric channel and the ATP binding catalytic channel. Casasnovas et. al. applied metadynamics and Markov state model to compute the kinetic rates of one inhibitor unbinding from $\mathrm{p} 38 \alpha$ with residence time of a few seconds. ${ }^{33}$ A systematic study of a series of inhibitors spanning a wide range of unbinding kinetics and exhibiting diverse structural features could provide further guidance to therapeutic design. However, it is quite challenging and even prohibited to compute the kinetic rates accurately for complex systems and slower unbinding processes with currently available methodologies and compute resources. As a result, few studies have been performed to compare the unbinding processes of different kinase inhibitors, especially in terms of kinetics. A recent computational study suggested unbinding pathways of p38 $\alpha$ 's typeI inhibitors SB2 and SK8, type-II inhibitor BIRB796, and type-III inhibitor LIG4 without ranking order by energetics and kinetics. ${ }^{34}$

Here, we report a computational study of two distinct p38 $\alpha$-BIRB796 analogs regarding their unbinding kinetics and pathways (Table 1). In the present study, we used MD simulation to study conformational dynamics and kinetics during dissociation processes of two drug-target complexes. We then performed metadynamics simulations to improve sampling of slow motions associated with ligand unbinding from $\mathrm{p} 38 \alpha$. The binding rates of two BIRB796 analogs are within the same order of magnitude, which simplifies the unbinding kinetic problem to a thermodynamic one. Our computational study provides a correct ranking order of the unbinding kinetics that is in agreement 
with the experimental results, i.e., slower unbinding of Lig8 relative to Lig3. ${ }^{27-28}$ Metadynamics simulation of p38 $\alpha$-ligand unbinding is most efficient using two CVs: ligand-cavity distance as $\mathrm{CV} 1$ and another geometric parameter as the second CV. The unbinding processes of the two ligands exhibit different FE landscapes of key motions. Slow motions during unbinding include relative orientation between the ligand and the protein, and a distance between the DFG motif and the cavity. Free energy landscape with DFG-cavity distance as CV indicates an inward motion of DFG motif occurring after dissociation of both ligands although they have different FE minima when bound. Rebinding of Lig8 to the secondary binding site during the dissociation process was revealed from the simulations, which is a plausible cause of prolonged residence time for Lig8. The fact of multiple unbinding pathways accessible for Lig3 indicates a higher probability and hence a faster rate of unbinding. Hydrophobic and hydrogen-bonding interactions between the ligand and the protein become unstable and are further disrupted upon unbinding while the binding site undergoes re-solvation. After the ligand unbinds from $\mathrm{p} 38 \alpha$, the hydrogen bond (H-Bond) network resets in the binding site, as shown in the unbound p38a structure. The embodied methods in this work can be considered as a general approach to understand unbinding kinetics and pathways of other kinase inhibitors of different kinase families. The novel mechanism of unbinding kinetics and multiple pathways revealed from this study can also potentially guide the kinase drug discovery.

Table 1. Kinetic and Thermodynamic Unbinding Parameters of p38 $\alpha$ inhibitors<smiles>[R]NC(=O)Nc1cc(C(C)(C)C)nn1[R2]</smiles> 


\begin{tabular}{|c|c|c|c|c|c|c|}
\hline Ligand & $\mathrm{R} 1$ & $\mathrm{R} 2$ & $\begin{array}{c}k_{\mathrm{on}} \\
\left(\mathrm{M}^{-1} \mathrm{~s}^{-1}\right)\end{array}$ & $\begin{array}{c}k_{\mathrm{off}} \\
\left(\mathrm{M}^{-1} \mathrm{~s}^{-1}\right)\end{array}$ & $\tau$ & $\begin{array}{c}K_{\mathrm{d}} \\
(\mathrm{nM})\end{array}$ \\
\hline 3 & & & $1.2 \times 10^{5}$ & $1.4 \times 10^{-1}$ & $7 \mathrm{~s}$ & 1160 \\
\hline 8 & & & $1.45 \times 10^{5}$ & $3.3 \times 10^{-3}$ & $5 \mathrm{~m}$ & 23 \\
\hline $\begin{array}{l}\text { BIRB- } \\
796\end{array}$ & & & $8.5 \times 10^{4}$ & $8.3 \times 10^{-6}$ & $33 \mathrm{~h}$ & 0.1 \\
\hline
\end{tabular}

Note: $\tau=1 / k_{\text {off }}$, refers to unbinding residence time. The values of $k_{\mathrm{on}}, k_{\mathrm{off}}$ and $K_{\mathrm{d}}$ are obtained from references. $^{27-28}$

\section{Results and Discussion}

\subsection{Selections of simulation models and slow motions}

Two lead compounds of p38 $\alpha$, Lig3 and Lig8 were chosen for this study considering their distinct structures and kinetic properties available from the experiments. As analogs of BIRB796 inhibitor, Lig3 and Lig8 exhibit the same magnitude of binding rates, therefore, the unbinding kinetic problem is simplified to be a thermodynamic one (Table 1). Like many other enhanced sampling methods such as steered MD,${ }^{35}$ temperature accelerated MD (TAMD), ${ }^{36}$ metadynamics involves sampling over suitable collective variables (CVs). ${ }^{37}$ Appropriate selection of CVs is not a trivial step, because overcoming hidden barriers for slow motions can be a significant challenge for CV-based free-energy calculations. Previous studies on $\mathrm{p} 38 \alpha$ MAPK provide us a wealth of biological information to compare the choices of $\mathrm{CVs}$ with a series of calculations. ${ }^{24-28}$ 
As a metadynamics simulation progresses, fluctuations of CVs become gradually enhanced to facilitate the escape from stable configurations of the system. Multiple sets of CV combinations were tested and led to different profiles of unbinding kinetics as listed in Table 2. Our study suggests that using only the distance between the ligand and the binding cavity on p38 $\alpha$ fails to describe the unbinding process properly, which has also been reported by others recently. ${ }^{33}$ Remarkably, for well-converged metadynamics FE simulations (Figure S1), the choice of two different sets of CVs lead to similar results in terms of thermodynamics and kinetics, which could be considered as a validation of our approach.

Table 2. Collective variables (CV) used in metadynamics simulations

\begin{tabular}{|c|l|l|l|}
\hline $\begin{array}{c}\text { Simulation } \\
\text { No. }\end{array}$ & \multicolumn{1}{|c|}{ Primary CV } & \multicolumn{1}{|c|}{ Secondary CV } & Unbinding \\
\hline 1 & Ligand-cavity distance (CV1) & N/A & N/A \\
\hline 2 & Ligand-cavity distance (CV1) & Ligand-cavity dihedral (CV2) & Lig3, Lig8 \\
\hline 3 & Ligand-cavity distance (CV1) & DFG-cavity distance (CV3) & Lig3, Lig8 \\
\hline 4 & Ligand-cavity distance (CV1) & L167-F169 torsion (CV4) & Lig3 \\
\hline 5 & Ligand-cavity distance (CV1) & Flap-catalytic site distance (CV5) & N/A \\
\hline
\end{tabular}

\subsection{Free energy landscape of inhibitor unbinding.}

Metadynamics simulations using all CV choices CV1-CV2, CV1-CV3, and CV1-CV4 led to consistent results, i.e., slower unbinding of Lig8 relative to Lig3 (Figure 2 4). The residence time of ligand unbinding can be defined as $\tau=1 / k_{\text {off }}$, where $k_{\text {off }}$ is the unbinding rate; the binding constant is defined as $K_{b}=k_{\mathrm{on}} / k_{\mathrm{off}}$, where $k_{\mathrm{on}}$ is the binding rate. Therefore, if inhibitors show similar binding rates, their residence time $\tau$ will be proportional to $K_{b}$, alternatively reciprocal to the dissociation constant $K_{d}, \tau \propto 1 / K_{d}$. In another words, stronger binder will bind for a longer time given the same order of magnitude of $k_{\mathrm{on}}$. The kinetic parameters have been reported for p38 $\alpha$ inhibitor BIRB796 
and its analogs. ${ }^{27-28}$ In Table 1, inhibitor BIRB796 and its analogs Lig3 and Lig8 all have experimental $k_{\text {on }}$ of about $1 \times 10^{5} \mathrm{M}^{-1} \mathrm{~s}^{-1}$, however, the three analogous molecules display quite diverse range of residence time from a few seconds to more than a day. In this case, the kinetic variation is solely determined by the binding affinity. Therefore, this unbinding kinetic problem can be reduced to a thermodynamic one.
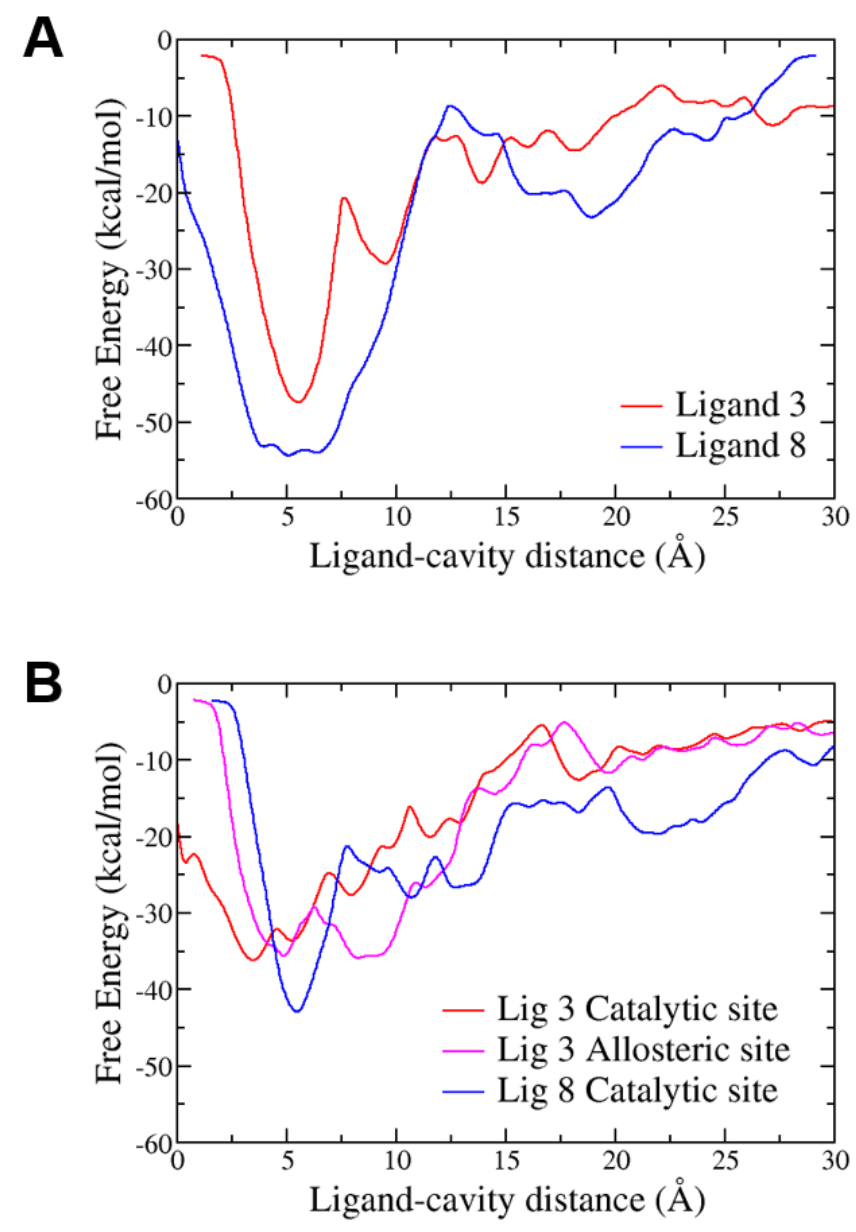

Figure 2. Free energy profiles of p38 $\alpha$ inhibitor unbinding. Unbinding FE profiles as a function of CV1 calculated from metadynamics simulation No. 2 (A) and No. 3 (B) respectively. For (B), Ligand 3 adopted different unbinding pathways, i.e., through the catalytic site (red) and allosteric site (margenta), while ligand 8 unbinds through the catalytic site (blue) pathway. 
Unbinding FE profile as a function of ligand-cavity distance calculated based on metadynamics simulations using CV1-CV2 (Figure 2A) suggests that Lig8 has a lower and broader global FE minimum compared to Lig3. Metadynamics simulations using CV1-CV3 (Figure 2B) also confirm that Lig8 has a lower global minimum at the binding cavity in comparison with Lig3. Free energy profiles confirm that Lig8 is a stronger binder of p38 $\alpha$ than Lig3. Given similar binding rates,${ }^{38} \mathrm{FE}$ results also indicate that Lig8 unbinds from p38 $\alpha$ more slowly than Lig3 does, which is consistent with the experiments. ${ }^{27-28}$

The converged FES reconstructed from metadynamics simulation reveals more information in terms of energetics corresponding to the fluctuation of CVs during the course of unbinding (Figure 3 and 4). To consider the relative orientation between the ligand and the protein, CV2 was defined according to the concept of dihedral angle in previous MD simulation study on ligandreceptor binding. ${ }^{10,39}$ Using ligand-cavity dihedral as a secondary $\mathrm{CV}$, unbinding free energy landscape of Lig8 is more complex than that of Lig3. The FE map demonstrates that Lig3 has a narrower FE minimum of about $-47 \mathrm{kcal} / \mathrm{mol}$ in the binding cavity when CV1 is about $6 \AA$ and $\mathrm{CV} 2$ is about $-140^{\circ}$ (Figure $2 \mathrm{~A}$ and $3 \mathrm{~A}$ ). By contrast, Lig8 has a broader binding FE minimum of about $-54 \mathrm{kcal} / \mathrm{mol}$ for Lig8 when the dihedral is about $-90^{\circ}$ with ligand-cavity distance spanning from $3 \AA$ to $8 \AA$ (Figure $2 \mathrm{~A}$ and $3 \mathrm{~A}$ ). Upon unbinding, Lig8 start to change its conformation and orientation relative to the protein while remain in the binding cavity with a CV1 of $\sim 5 \AA$. Using DFG-cavity distance as the second CV, the FES exhibits similar characteristics in metadynamics simulations with CV1-CV3, as in simulations with CV1-CV2 (Figure 4). The binding of type II inhibitors Lig3 and Lig8 both lead to outward orientation of DFG motif. The DFG-cavity distance (CV3) fluctuates between $8 \AA$ and $12 \AA$ when Lig3 bound and increases to $11 \sim 14 \AA$ when Lig8 is in the cavity. 

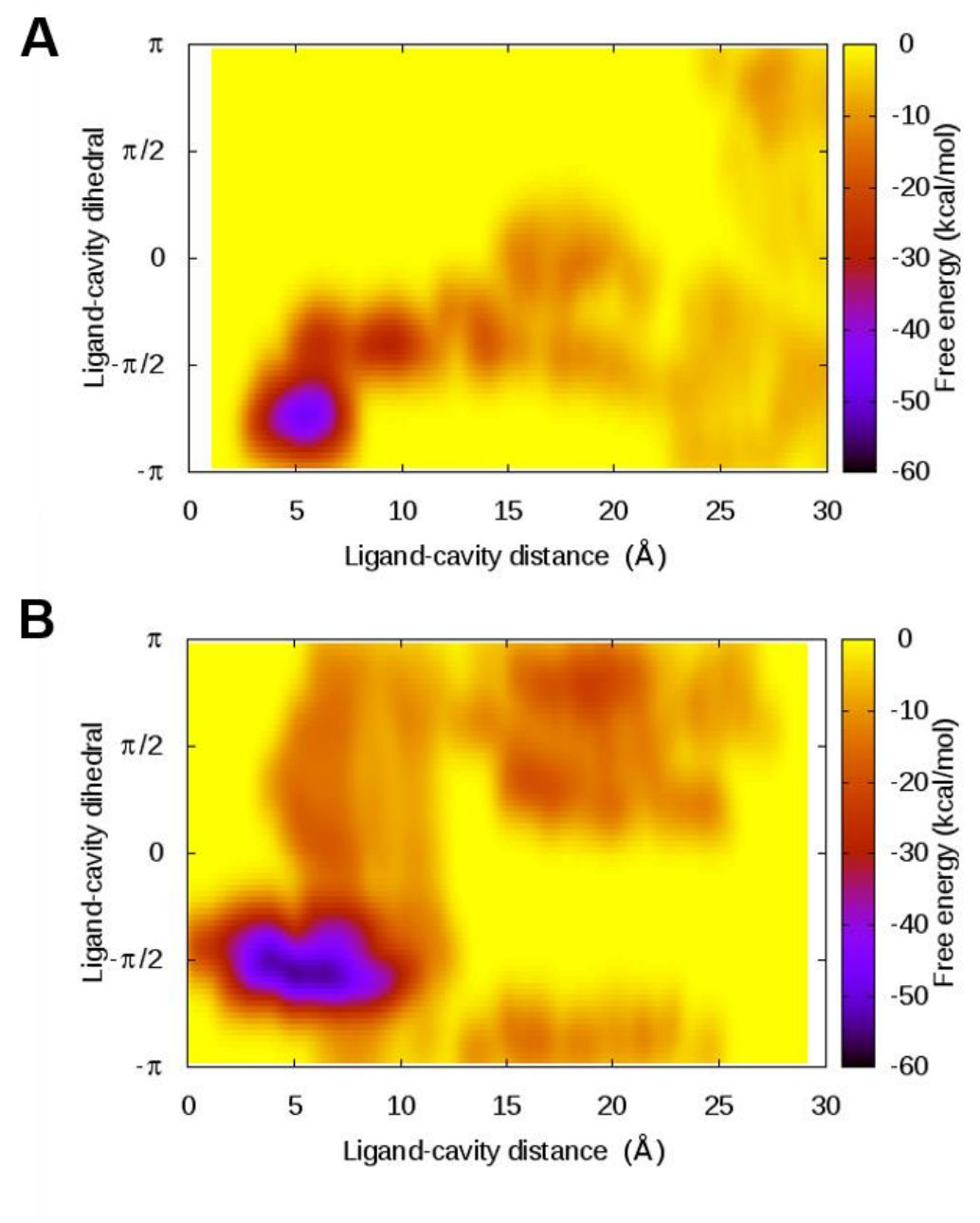

Figure 3. Free energy surfaces of $\mathrm{p} 38 \alpha$ inhibitor unbinding calculated from metadynamics simulations (No. 2) using CV1 and CV2 for ligand 3 (A) and ligand 8 (B). 


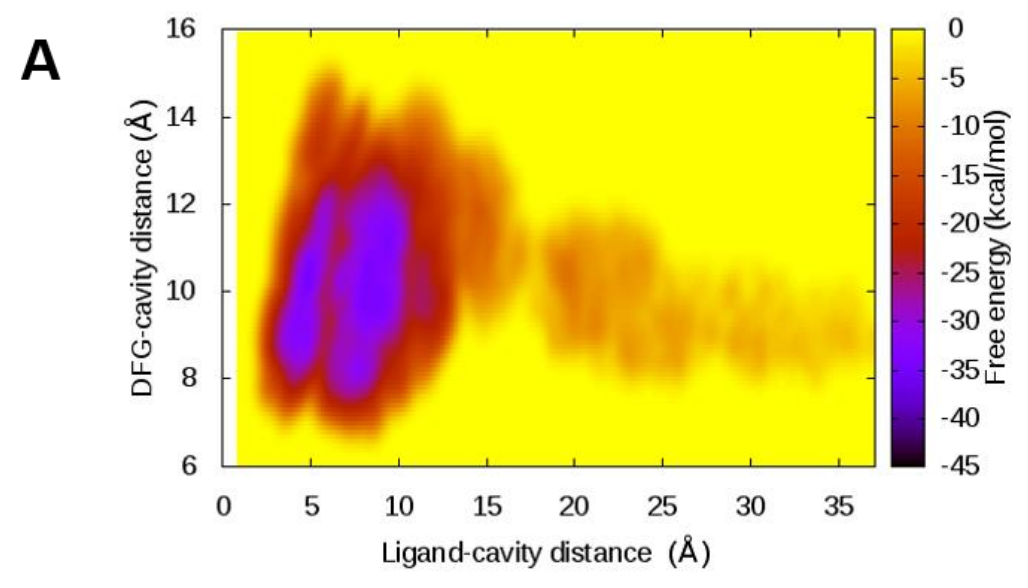

B
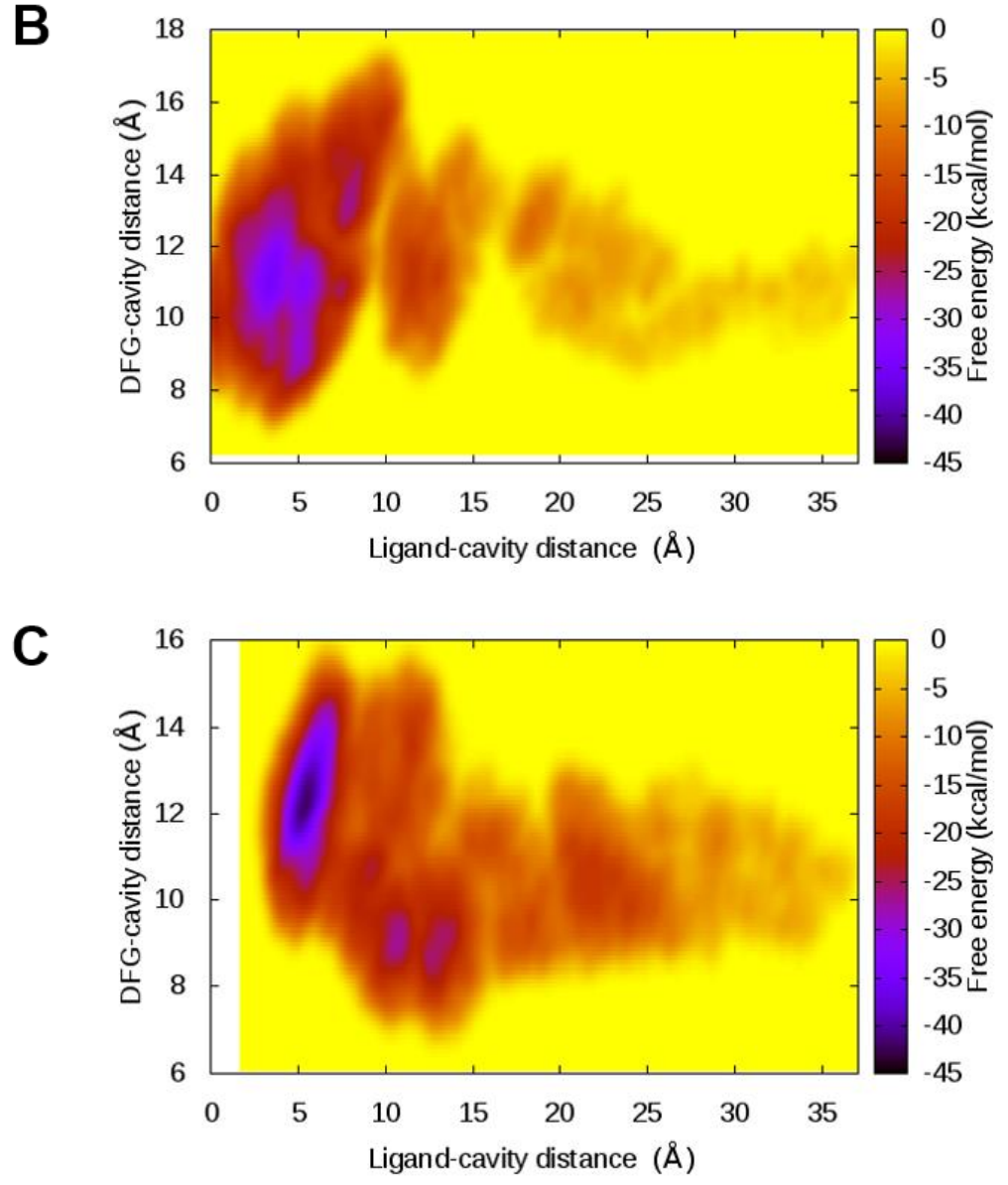

Figure 4. Free energy surfaces of $\mathrm{p} 38 \alpha$ inhibitor unbinding calculated from metadynamics simulations (No. 3) using CV1 and CV3. Ligand 3 unbinds through the allosteric site (A) or the catalytic site (B) and, ligand 8 unbinds through the catalytic site (C). 


\subsection{Unbinding kinetics and rebinding.}

In the equilibrated configuration, the distance between the inhibitor and the cavity fluctuates between $5 \AA$ and $10 \AA$ (Figure 5 7), the inhibitor is considered fully unbound when CV1 is greater than $20 \AA$. For simulation using CV1-CV2, Lig3 unbinding starts at $\sim 1$ ns (Figure 5A and 5C), while for Lig8, unbinding occurs after $2.5 \mathrm{~ns}$ followed with rebinding at $3.5 \mathrm{~ns}$ before complete dissociation (Figure 5B and 5D).
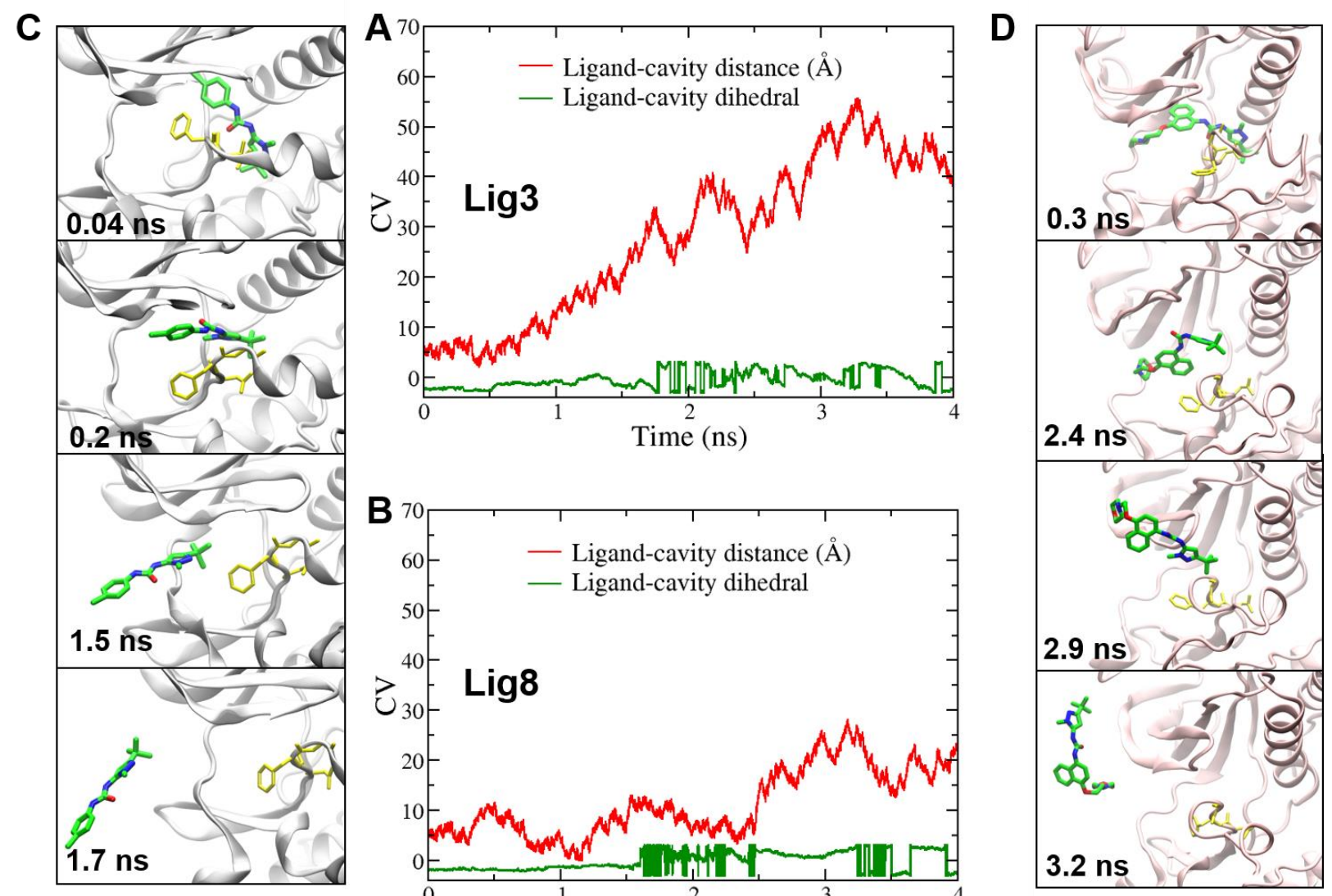

Figure 5. Metadynamics simulations using CV1 and CV2. Time series of collective variables CV1 (red) and CV2 (green) are shown for ligand 3 (A) and ligand 8 (B) with representative snapshots taken from the trajectories for ligand $3(\mathrm{C})$ and ligand 8 (D) respectively. In (C) and (D), snapshot conformations are shown for p38 (cartoon), the ligand (colored licorice) and nonhydrogen atoms of DFG loop (yellow licorice). The images of snapshot conformations were created with $\mathrm{VMD}^{40}$ 
In metadynamics simulations using CV1-CV3, Lig3 unbinding occurs after 6 ns (Figure 6A and 7A), while for Lig8, this occurs at about $6 \mathrm{~ns}$ and the ligand rebinds at $7.5 \mathrm{~ns}$ before complete dissociation from the protein (Figure 6B and 6D). In the meantime, the protein exhibits different FE landscapes of key motions during unbinding. Rebinding to the secondary binding site underneath the glycine-rich phosphate-binding loop (P-loop) also potentially contributes to the longer residence time of Lig8 (Figure 5B and 6B). The R1 group ethoxy-morpholine-naphthalene on Lig8 is bulkier than the chlorophenyl ring on Lig3 (Table 1). The naphthalene ring is more lipophilic than phenyl ring, while morpholine could form H-Bond with nearby Met109. ${ }^{38}$ All these interactions strengthen the binding affinity and decrease the unbinding rate of Lig8. Lig8 rebinds to $338 \alpha$ surface through interaction between this functional moiety and Val30 and Val38 side chain on the P-loop (Figure 5D and 6D). The rebinding of Lig8 leads to prolonged apparent target occupancy, and consequently longer residence time. 


\section{C}

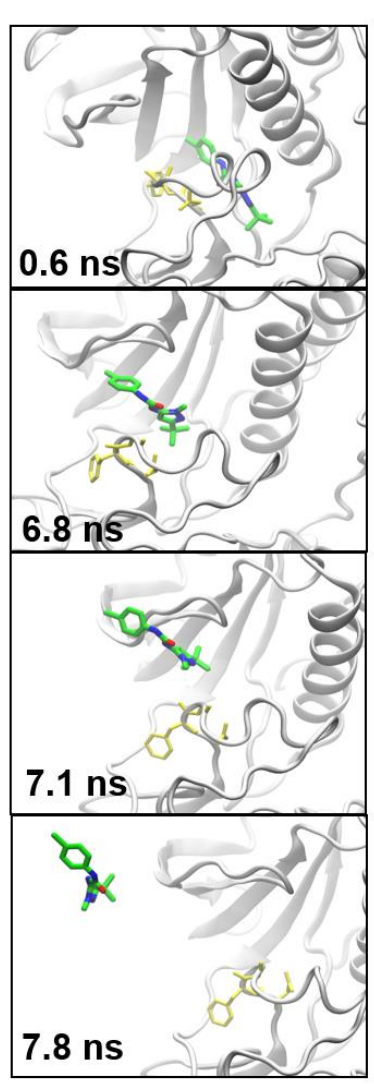

A 70

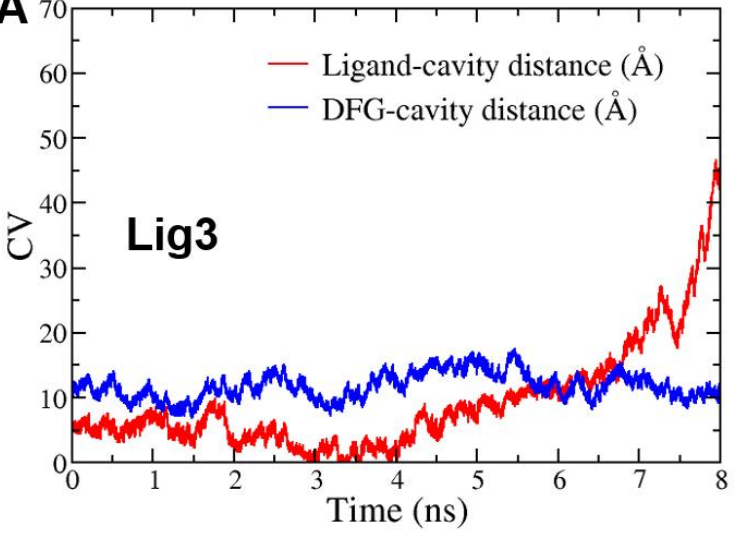

B

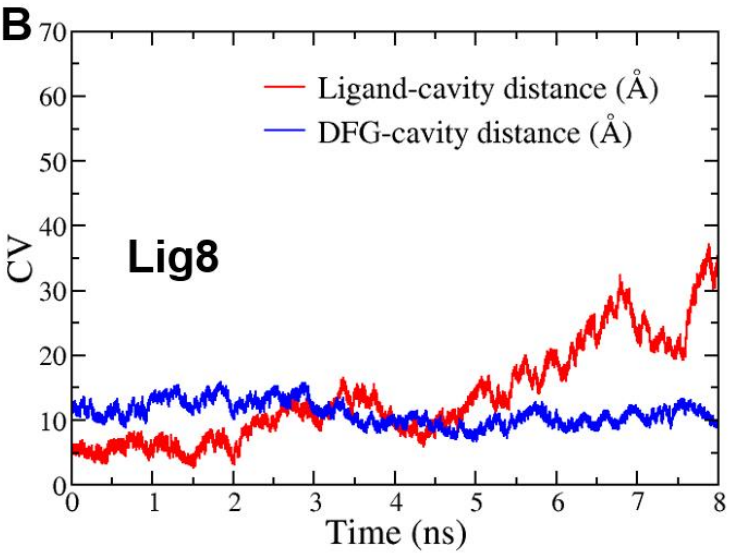

D

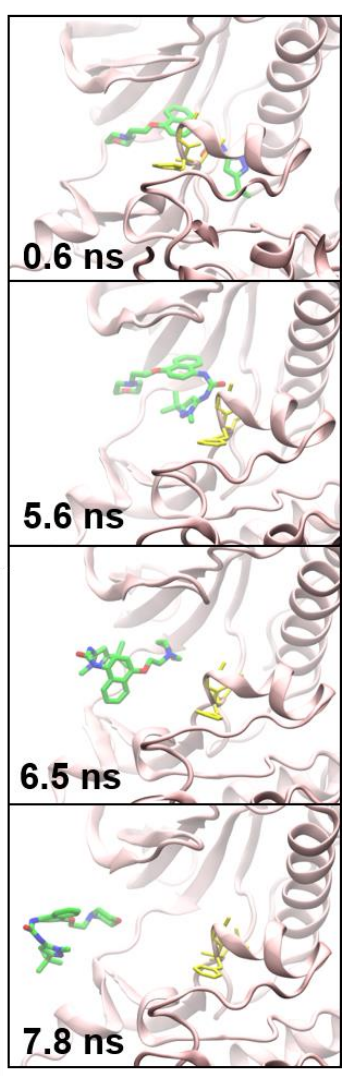

Figure 6. Metadynamics simulations using CV1 and CV3. Time series of collective variables CV1 (red) and CV3 (blue) are shown for ligand 3 (A) and ligand 8 (B) with representative snapshots taken from the trajectories for ligand $3(\mathrm{C})$ and ligand 8 (D) respectively. 
C

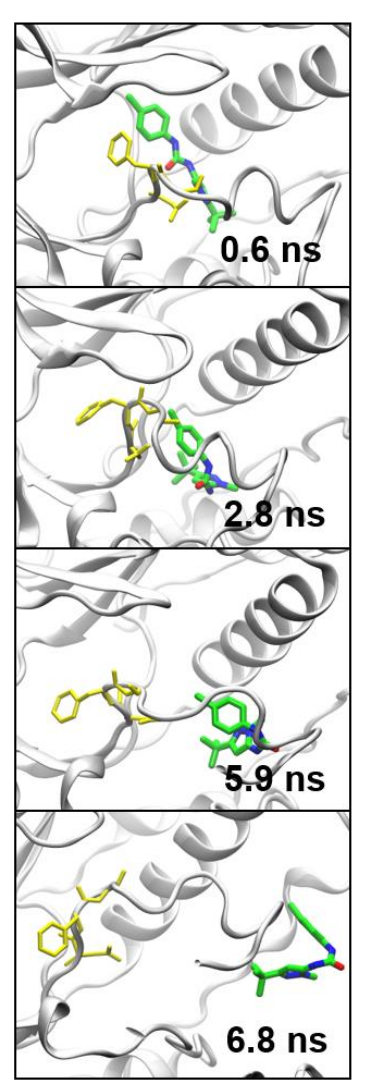

A

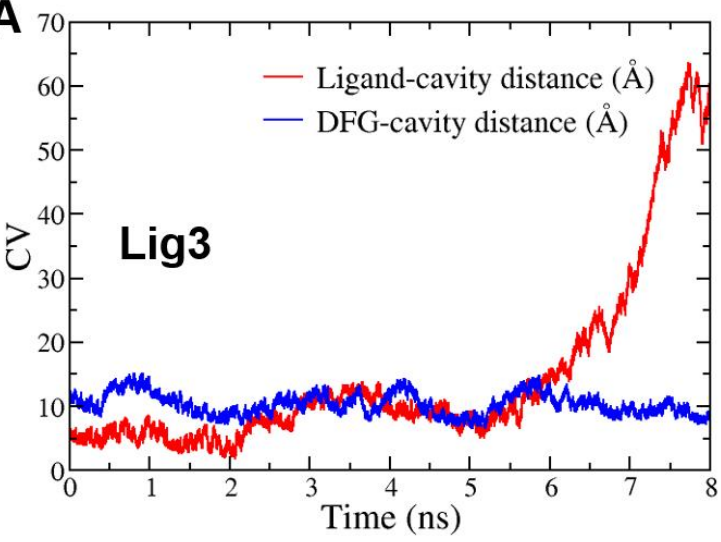

B

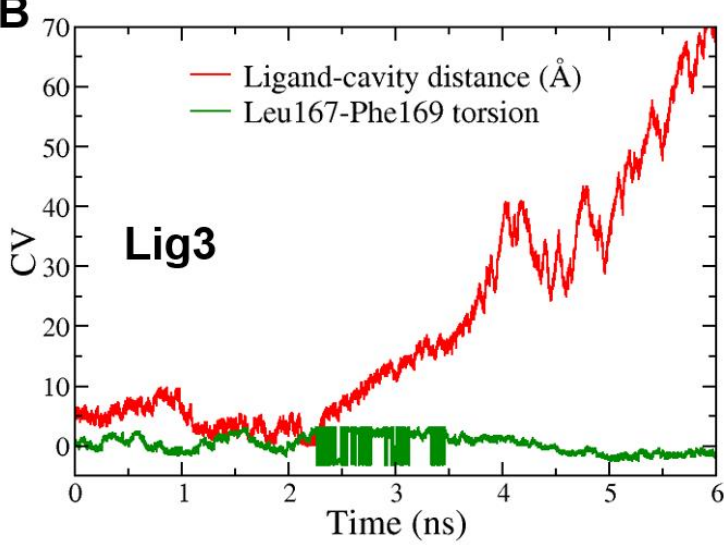

D

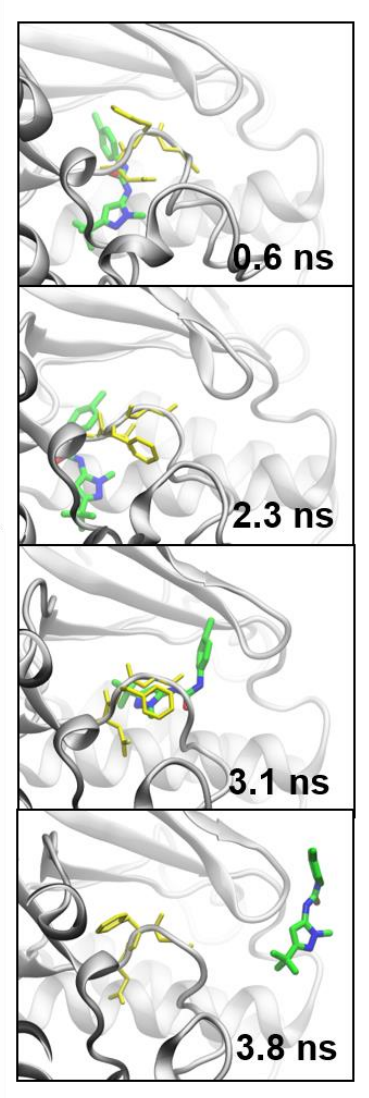

Figure 7. The alternative unbinding pathways for ligand 3. Time series of collective variables for metadynamics simulations using CV1-CV3 (A) and CV1-CV4 (B) with representative snapshots taken from trajectories using CV1-CV3 (C) and CV1-CV4 (D) respectively. (A) and (C) represent unbinding through the allosteric site, while (B) and (D) suggest the third unbinding pathway from the P-loop. The CVs in time series are colored in red, blue and green for CV1, CV3 and CV4 respectively.

\subsection{Multiple unbinding pathways.}

In this study, metadynamics simulations of Lig8 unbinding using different sets of CVs result in a single pathway through the ATP-binding site, also known as the catalytic channel (Figure 5D and 6D). ${ }^{32}$ By contrast, Lig3 unbinding exhibits three different unbinding pathways: 1) through the catalytic site (Figure 5C and 6C), 2) through the allosteric site (Figure 7C), and 3) opening the glycine-rich P-loop (Figure 7D). The common feature of Lig3 unbinding through the catalytic 
pathway and the flap pathway is that inhibitors exit the binding site with their R1 aromatic-ring group exposed to the solvent first (Figure 5D, 6D and 7D). In comparison, Lig3 unbinding through the allosteric pathway requires complex conformational changes (Figure 7C). During the simulation, Lig3 became unstable at $2.8 \mathrm{~ns}$, and then oriented itself into a bended conformation and began to egress the binding cavity at $5.9 \mathrm{~ns}$, finally fully escaped the receptor at $6.8 \mathrm{~ns}$ (Figure 7C).

The correlation of DFG motion with unbinding suggests that CV3, the distance from DFG motif to cavity, is a slow motion relevant to the unbinding through the allosteric pathway. There exists a higher energy barrier for Lig8 to escape the allosteric site. Multiple unbinding pathways available for Lig3 lead to a higher probability and hence a faster rate of unbinding, which suggests a novel mechanism of unbinding kinetics for $\mathrm{p} 38 \alpha$ inhibitors.

\subsection{The DFG motif motion.}

The DFG loop regulates kinase activation by altering side chain conformation. ${ }^{22}$ The kinase become inactive by adopting an DFG-out conformation when type II inhibitors are bound. ${ }^{27}$ It was also reported that the backbone of DFG motif functions as a hydrophobic core to interact with the t-butyl tail of the inhibitors. ${ }^{33}$ From metadynamics simulations using CV1-CV3, we found that for both inhibitors, the DFG-cavity distance is shortened by $5 \sim 10 \AA$ after the inhibitor unbinding. This ligand-free conformation mimics the active conformation of an apo kinase. Remarkably, before dissociation of ligands, a quick inward motion of $\sim 5 \AA$ were also observed for DFG motif relative to the cavity (Figure 6A, 6B and 7A). Then facilitated by the DFG outward motion, the cavity opened to allow the ligand to exit, as indicated by the increase of DFG-cavity distance (10 $\AA$ to $\sim 15 \AA$ A). After ligand dissociation, DFG-cavity distance was shortened to $\sim 10 \AA$. Therefore, FE 
landscape with the DFG-cavity distance as the second CV suggests that the dynamic motion of DFG motif is coupled with the dissociation of both ligands, even though they have different FE minima when bound.

\subsection{The Leu167-Phe169 side chain torsion change.}

It was reported that the side chain opening of Phe169 accompanies with BIRB796 inhibitor binding. ${ }^{38}$ During 6 ns metadynamics simulation with the second CV defined by LF side chain torsion Leu167C $\beta$-Leu167C $\alpha$-Phe169C $\alpha$-Phe169C $\gamma$ (CV4), only Lig3 unbinds at 3 ns, Lig8 remains bound throughout 6 ns simulations (Figure 7B and Figure S2). The kinetic difference implies that using the LF side chain torsion is not sufficient to characterize the unbinding process of Lig8. Therefore, CV4 is not an appropriate choice of the second CV. This demonstrates the nature of complex conformation dynamics controlling the unbinding kinetics.

Electrostatic and hydrophobic interactions stabilize ligand-binding to p38 $\alpha$ binding site. Residue Lys53, Asp168 and Arg173 form H-Bonds with water in the binding site when Lig3 is bound (Figure S3 top). Two urea amide groups of the ligand form H-Bonds with the side chain carboxylate group of Glu71. Upon Lig3 dissociation, H-Bond interactions between the ligand and the protein become unstable and further disrupted (Figure S3 middle). In the meantime, the binding site undergoes re-solvation. After Lig3 unbinds from p38a, the H-Bond network resets in the binding site (Figure S3 bottom), resembling the ligand-unbound p38 $\alpha$ structure.

By comparing the ligand structures in Table 1, all three compounds have a common scaffold of a pyrazole ring attached to a urea group. Lig3 bears a benzenyl chloride at R1 position, while 
Lig8 bears an ethoxy morpholin attached to naphthalene at R1 position, rendering extra hydrophobicity to bind p $38 \alpha$ more strongly in the hydrophobic cavity underneath the P-loop.

\subsection{The P-loop (Flap) motion.}

A glycine-rich motif on the P-loop forms a flexible flap at the catalytic site. ${ }^{41}$ To monitor the open-and-close motion of the flap and characterize its relationship with inhibitor unbinding, another collective variable CV5 was defined as a distance between $\mathrm{C} \alpha$ atoms of Ser32, Gly33, Ala34 and Thr35 and C $\alpha$ atoms of the catalytic site residues Asn155, Leu156, and Ala157. However, neither Lig3 nor Lig8 is able to disassociate from p38 $\alpha$ within 8 ns of metadynamics simulations using CV1 and CV5 (Figure S4). Therefore, the flap motion is not a slow motion directly related to ligand unbinding kinetics.

Although current study generates correct rank order of binding affinities and unbinding rates for the two ligands, a fairly large discrepancy of the absolute FE values remains an issue. As a fast-developing CV-biasing method, the limitation of metadynamics-based methods should not be neglected. When using these methods, one should pay more attention on the efficiency and convergence, especially potential mis-interpretation of converged FES calculation over suboptimized reaction coordinate. ${ }^{42}$ Nevertheless, for such kind of systems with high therapeutic values, future study should be focused on optimizing CVs and applying more efficient and comprehensive algorithm to the sampling.

\section{Materials and Methods}

\subsection{System setup.}


The kinetics of BIRB796 analogs binding to p38 $\alpha$ MAPK have been measured experimentally as listed in Table $1 .^{27-28}$ High-resolution 3D structures of p38 $\alpha$ MAPK are abundant in the Protein Data Bank. Crystal structure of p38 $\alpha$ MAPK in complex with Lig3 (PDB code 1KV1) was used for the initial conformation of the protein. All simulation models were constructed as monomers encompassing residue number 5 to 352 . Missing residues in the flap and the activation loop were added using the FALC-Loop server. ${ }^{43}$ The loop conformations were generated by fragment assembly and analytical loop closure. The model p38 $\alpha$ MAPK in complex with Lig8 was built by modifying the ligand structure in $1 \mathrm{KV} 1$.

\subsection{Equilibration with MD simulation}

All simulations were carried out using NAMD 2.8 package $^{44}$ patched with PLUMED $1.3^{45}$. CHARMM36 all-atom force field ${ }^{46}$ was used for protein and salt ions. CGenFF force field parameters for BIRB796 analogs were created with ParamChem and validated using Gaussian09. ${ }^{47}$ The systems were first solvated with a box of about 17500 TIP3P water molecules, ${ }^{48}$ neutralized with $56 \mathrm{Na}+$ and $49 \mathrm{Cl}$ - ions to reproduce the ionic strength of the experiments. ${ }^{28}$ Periodic Boundary Condition was applied throughout the simulation with a box size of $102 \times 80 \times 76 \AA^{3}$. While keeping protein nonhydrogen atoms fixed, ions and hydrogens were first relaxed by performing 1000 steps of conjugate gradient energy minimization. Then with protein backbone atoms fixed, the systems were relaxed by performing 500 steps of minimization. The system was then subjected to 500 steps of minimization and 20 ps heating with the protein restrained with a 1 $\mathrm{kcal} / \mathrm{mol} / \AA^{2}$ harmonic force constant. After removal of harmonic constraints, the system underwent Langevin dynamics with Nose-Hoover barostat ${ }^{49-50}$ at constant pressure of 1.0132 bar and $298 \mathrm{~K}$ temperature. A cutoff of $12 \AA$ and a switching distance of $10 \AA$ were applied for non- 
bonded interactions. Electrostatics was evaluated with the particle mesh Ewald method. The SETTLE algorithm ${ }^{51}$ was used to constrain all bonds involving hydrogen atoms.

\subsection{Metadynamics simulations of unbinding.}

The equilibrated structures were used as starting points for metadynamics simulation. Geometrical collective variables (CV) were defined to characterize the unbinding pathway, as listed in Table 2. Specifically, CV1 is the distance between the centroid of the central diaryl urea group on the ligand and the centroid of $\mathrm{C} \alpha$ atoms of binding cavity residues Leu74, Leu104, and Gly137; CV2 is the dihedral angle between two major inertia axes of the ligand and the protein, identified by two centroids on the ligand (the central diaryl urea group and benzenyl group) and centroids of two sets of $\mathrm{C} \alpha$ atoms in the stable $\alpha$-helices composed of residue number 131-140 and 204-213).

To study the key motions of the activation loop during ligand dissociation from the protein, additional geometrical CVs were used. CV3 was defined as the distance between the centroid of three C $\alpha$ atoms of the DFG motif (Asp168, Phe169 and Gly170) and the centroid of three C $\alpha$ atoms of binding site residues Leu74, Leu104, and Gly137. CV4 were defined to characterize the side chain motion of Phe169 on the DFG motif, i.e., torsion angle Leu167C $\beta$-Leu167C $\alpha$-Phe169C $\alpha$ Phe169C $\gamma$.

To monitor the open-and-close motion of the flap, a distance CV5 was defined between the centroid of four C $\alpha$ atoms of Ser32, Gly33, Ala34 and Thr35 and the centroid of three C $\alpha$ atoms of the catalytic site residues Asn155, Leu156, and Ala157. Using the equilibrated structures, two independent runs of $8 \mathrm{~ns}$ simulations were carried out using each of the five CV sets for both 
ligands (Table 2), with a total of 20 simulations. The multi-dimensional unbinding FE landscapes were reconstructed based on the Gaussians with optimal height and width. In all simulations, the Gaussian-shaped potentials were deposited every 100 simulation steps with an initial height of 0.1 $\mathrm{kcal} / \mathrm{mol}$. The Gaussian width was set to $0.35 \AA$ for ligand-cavity distance, $0.25 \AA$ for DFG-cavity distance and flap-catalytic site distance, and 0.35 for ligand-cavity dihedral and Leu167-Phe169 torsion angle. The convergence of the metadynamics simulations was monitored and ensured by calculating the estimate of FES with an interval of every 100 Gaussians deposited.

\section{Conclusion}

In the present study, metadynamics simulations were performed to enhance sampling of slow motions associated with ligand unbinding from p38 $\alpha$ MAPK. Two analogs of BIRB796 inhibitor share the same magnitude of binding rates, therefore, the unbinding kinetic problem was simplified to be a thermodynamic one. Current study of type II inhibitors confirms slower unbinding of Lig8 relative to Lig3. Metadynamics simulation of p38 $\alpha$-ligand unbinding is most efficient using two CVs: the ligand-cavity distance as CV1 and another geometric parameter as the second CV. The unbinding kinetics involves different FE landscapes of the key motions. Slow motions related to unbinding are relative orientation between the ligand and the protein, and the distance from the DFG motif to the cavity.

FE profiles confirm that Lig8 binds to p38 $\alpha$ more strongly than Lig3. The unbinding pathway of Lig8 is only found to be initiated from the catalytic site; rebinding of Lig8 to the secondary binding site underneath the P-loop may contribute to the longer residence time. The R1 group ethoxy-morpholin-naphthalene group on Lig8 is bulkier than the chlorophenyl ring on Lig3, providing extra hydrophobic and H-Bond interactions with the cavity, and consequently strengthen 
the binding affinity and decrease the unbinding rate of Lig8. Lig8 rebinds to p38 $\alpha$ surface through interaction between this functional moiety and Val30 and Val38 side chain on the P-loop. Other than the previously reported two unbinding pathways, the catalytic channel and the allosteric channel, ${ }^{34}$ an additional unbinding pathway of Lig3 underneath the P-loop were revealed. Multiple unbinding pathways available for Lig3 lead to a higher probability and hence a faster rate of unbinding, which suggests an underlying mechanism of unbinding kinetics for p38 $\alpha$ inhibitors.

According to FES with DFG-cavity distance as the second CV, inward motion of DFG motif occurs upon dissociation of both ligands, albeit with different FE minima in ligand-bound state.

Although the P-loop in the p38 catalytic site is important for ligand unbinding, the flap motion itself is not a slow motion that is directly associated with unbinding. Hydrophobic and $\mathrm{H}-$ Bond interactions between the ligand and protein become unstable and further disrupted upon unbinding while the binding site undergoes re-solvation. After the ligand unbinds from $\mathrm{p} 38 \alpha$, the H-Bond network is recovered in the binding site, as shown in the unbound p38 $\alpha$ structure.

To summarize, the approach utilized in our study can be applied to understand unbinding kinetic and pathway of other kinase inhibitors even for different kinase families. The kinetic information obtained from this study can potentially guide the kinase inhibitor design.

Acknownledgments: This work was partly supported by Grant Numbers R01LM011986 from the National Library of Medicine (NLM), R01GM122845 from the National Institute of General Medical Sciences (NIGMS), and R01AD057555 of National Institute of Aging of the National Institute of Health (NIH). The authors acknowledge the compute resources and support provided by CUNY High Performance Computing Center (HPCC). The CUNY HPCC is operated by the 
College of Staten Island and funded, in part, by grants from the City of New York, State of

New York, CUNY Research Foundation, and National Science Foundation Grants CNS-

0958379, CNS-0855217 and ACI 1126113.

Conflicts of Interest: The authors declare no conflict of interest.

\section{REFERENCES}

1. Copeland, R. A., Conformational adaptation in drug-target interactions and residence time. Future Med Chem 2011, 3 (12), 1491-1501.

2. Lu, H.; Tonge, P. J., Drug-target residence time: critical information for lead optimization. Curr Opin Chem Biol 2010, 14 (4), 467-474.

3. Copeland, R. A., The drug-target residence time model: a 10-year retrospective. Nat Rev Drug Discov 2016, 15 (2), 87-95.

4. $\quad$ Spagnuolo, L. A.; Eltschkner, S.; Yu, W. X.; Daryaee, F.; Davoodi, S.; Knudson, S. E.; Allen, E. K. H.; Merino, J.; Pschibul, A.; Moree, B.; Thivalapill, N.; Truglio, J. J.; Salafsky, J.; Slayden, R. A.; Kisker, C.; Tonge, P. J., Evaluating the Contribution of Transition-State Destabilization to Changes in the Residence Time of Triazole-Based InhA Inhibitors. J Am Chem Soc 2017, 139 (9), 3417-3429.

5. Callegari, D.; Lodola, A.; Pala, D.; Rivara, S.; Mor, M.; Rizzi, A.; Capelli, A. M., Metadynamics Simulations Distinguish Short- and Long-Residence-Time Inhibitors of CyclinDependent Kinase 8. Journal of Chemical Information and Modeling 2017, 57 (2), 159-169.

6. Singh, J.; Petter, R. C.; Baillie, T. A.; Whitty, A., The resurgence of covalent drugs. Nat Rev Drug Discov 2011, 10 (4), 307-317.

7. Bradshaw, J. M.; McFarland, J. M.; Paavilainen, V. O.; Bisconte, A.; Tam, D.; Phan, V. T.; Romanov, S.; Finkle, D.; Shu, J.; Patel, V.; Ton, T.; Li, X. Y.; Loughhead, D. G.; Nunn, P. A.; Karr, D. E.; Gerritsen, M. E.; Funk, J. O.; Owens, T. D.; Verner, E.; Brameld, K. A.; Hill, R. J.; Goldstein, D. M.; Taunton, J., Prolonged and tunable residence time using reversible covalent kinase inhibitors. Nat Chem Biol 2015, 11 (7), 525-+.

8. Chiu, S. H.; Xie, L., Toward High-Throughput Predictive Modeling of Protein Binding/Unbinding Kinetics. Journal of Chemical Information and Modeling 2016, 56 (6), 11641174.

9. Dror, R. O.; Dirks, R. M.; Grossman, J. P.; Xu, H. F.; Shaw, D. E., Biomolecular Simulation: A Computational Microscope for Molecular Biology. Annu Rev Biophys 2012, 41, 429-452.

10. Ge, X. X.; Roux, B., Absolute Binding Free Energy Calculations of Sparsomycin Analogs to the Bacterial Ribosome. J Phys Chem B 2010, 114 (29), 9525-9539.

11. Sugita, Y.; Okamoto, Y., Replica-exchange molecular dynamics method for protein folding. Chemical Physical Letter 1999, 314 (1-2), 141-151. 
12. Hamelberg, D.; Mongan, J.; McCammon, J. A., Accelerated molecular dynamics: a promising and efficient simulation method for biomolecules. J Chem Phys 2004, 120 (24), 1191929.

13. Torrie, G. M. V., J. P., Nonphysical Sampling Distributions in Monte Carlo Free-Energy Estimation: Umbrella Sampling. J Comput Phys 1977, 23 (2), 187-199.

14. Isralewitz, B.; Baudry, J.; Gullingsrud, J.; Kosztin, D.; Schulten, K., Steered molecular dynamics investigations of protein function. J Mol Graph Model 2001, 19 (1), 13-25.

15. Laio, A.; Parrinello, M., Escaping free-energy minima. P Natl Acad Sci USA 2002, 99 (20), 12562-12566.

16. Barducci, A.; Bonomi, M.; Parrinello, M., Metadynamics. Wires Comput Mol Sci 2011, 1 (5), 826-843.

17. Valsson, O.; Tiwary, P.; Parrinello, M., Enhancing Important Fluctuations: Rare Events and Metadynamics from a Conceptual Viewpoint. Annu Rev Phys Chem 2016, 67, 159-184.

18. Deganutti, G.; Moro, S., Estimation of kinetic and thermodynamic ligand-binding parameters using computational strategies. Future Med Chem 2017, 9 (5), 507-523.

19. Bernetti, M.; Masetti, M.; Rocchia, W.; Cavalli, A., Kinetics of Drug Binding and Residence Time. Annu Rev Phys Chem 2019.

20. Tiwary, P.; Parrinello, M., From Metadynamics to Dynamics. Phys Rev Lett 2013, 111 (23).

21. Tiwary, P.; Limongelli, V.; Salvalaglio, M.; Parrinello, M., Kinetics of protein-ligand unbinding: Predicting pathways, rates, and rate-limiting steps. P Natl Acad Sci USA 2015, 112 (5), E386-E391.

22. Huse, M.; Kuriyan, J., The conformational plasticity of protein kinases. Cell 2002, 109 (3), 275-82.

23. Zarubin, T.; Han, J. H., Activation and signaling of the p38 MAP kinase pathway. Cell Res 2005, 15 (1), 11-18.

24. Igea, A.; Nebreda, A. R., The Stress Kinase p38 alpha as a Target for Cancer Therapy. Cancer Res 2015, 75 (19), 3997-4002.

25. Kumar, S.; Boehm, J.; Lee, J. C., p38 map kinases: Key signalling molecules as therapeutic targets for inflammatory diseases. Nat Rev Drug Discov 2003, 2 (9), 717-726.

26. Martin, E. D.; Bassi, R.; Marber, M. S., p38 MAPK in cardioprotection - are we there yet? Brit J Pharmacol 2015, 172 (8), 2101-2113.

27. Pargellis, C.; Tong, L.; Churchill, L.; Cirillo, P. F.; Gilmore, T.; Graham, A. G.; Grob, P. M.; Hickey, E. R.; Moss, N.; Pav, S.; Regan, J., Inhibition of p38 MAP kinase by utilizing a novel allosteric binding site. Nat Struct Biol 2002, 9 (4), 268-272.

28. Regan, J.; Capolino, A.; Cirillo, P. F.; Gilmore, T.; Graham, A. G.; Hickey, E.; Kroe, R. R.; Madwed, J. R.; Moriak, M.; Nelson, R.; Pargellis, C. A.; Swinamer, A.; Torcellini, C.; Tsang, M.; Moss, N., Structure-activity relationships of the p38 alpha MAP kinase inhibitor 1-(5-tertButyl-2-p-tolyl-2H-pyrazol-3-yl)-3-[4-(2-morpholin-4-yl-ethoxy)naphthalen-1-yl]urea (BIRB 796). J Med Chem 2003, 46 (22), 4676-4686.

29. The PyMOL Molecular Graphics System, Version 1.7.2.1. Schrödinger, LLC.

30. Wang, Z.; Canagarajah, B. J.; Boehm, J. C.; Kassisa, S.; Cobb, M. H.; Young, P. R.; AbdelMeguid, S.; Adams, J. L.; Goldsmith, E. J., Structural basis of inhibitor selectivity in MAP kinases. Structure 1998, 6 (9), 1117-28.

31. Yang, Y.; Shen, Y. L.; Liu, H. X.; Yao, X. J., Molecular Dynamics Simulation and Free Energy Calculation Studies of the Binding Mechanism of Allosteric Inhibitors with p38 alpha MAP Kinase. Journal of Chemical Information and Modeling 2011, 51 (12), 3235-3246. 
32. Sun, H. Y.; Tian, S.; Zhou, S. Y.; Li, Y. Y.; Li, D.; Xu, L.; Shen, M. Y.; Pan, P. C.; Hou, T. J., Revealing the favorable dissociation pathway of type II kinase inhibitors via enhanced sampling simulations and two-end-state calculations. Sci Rep-Uk 2015, 5.

33. Casasnovas, R.; Limongelli, V.; Tiwary, P.; Carloni, P.; Parrinello, M., Unbinding Kinetics of a p38 MAP Kinase Type II Inhibitor from Metadynamics Simulations. J Am Chem Soc 2017, 139 (13), 4780-4788.

34. You, W.; Chang, C. A., Role of Molecular Interactions and Protein Rearrangement in the Dissociation Kinetics of p38alpha MAP Kinase Type-I/II/III Inhibitors. J Chem Inf Model 2018, 58 (5), 968-981.

35. Isralewitz, B.; Gao, M.; Schulten, K., Steered molecular dynamics and mechanical functions of proteins. Curr Opin Struct Biol 2001, 11 (2), 224-30.

36. Maragliano, L.; Vanden-Eijnden, E., A temperature accelerated method for sampling free energy and determining reaction pathways in rare events simulations. Chem Phys Lett 2006, 426 (1-3), 168-175.

37. Abrams, C.; Bussi, G., Enhanced Sampling in Molecular Dynamics Using Metadynamics, Replica-Exchange, and Temperature-Acceleration. Entropy-Switz 2014, 16 (1), 163-199.

38. Regan, J.; Pargellis, C. A.; Cirillo, P. F.; Gilmore, T.; Hickey, E. R.; Peet, G. W.; Proto, A.; Swinamer, A.; Moss, N., The kinetics of binding to p38 MAP kinase by analogues of BIRB 796. Bioorg Med Chem Lett 2003, 13 (18), 3101-3104.

39. Ge, X. X.; Roux, B., Calculation of the standard binding free energy of sparsomycin to the ribosomal peptidyl-transferase P-site using molecular dynamics simulations with restraining potentials. J Mol Recognit 2010, 23 (2), 128-141.

40. Humphrey, W.; Dalke, A.; Schulten, K., VMD: Visual molecular dynamics. J Mol Graph Model 1996, 14 (1), 33-38.

41. Gomez-Gutierrez, P.; Rubio-Martinez, J.; Perez, J. J., Identification of Potential Small Molecule Binding Pockets in p38alpha MAP Kinase. J Chem Inf Model 2017, 57 (10), 2566-2574. 42. Cavalli, A.; Spitaleri, A.; Saladino, G.; Gervasio, F. L., Investigating Drug-Target Association and Dissociation Mechanisms Using Metadynamics-Based Algorithms. Accounts Chem Res 2015, 48 (2), 277-285.

43. Ko, J.; Lee, D.; Park, H.; Coutsias, E. A.; Lee, J.; Seok, C., The FALC-Loop web server for protein loop modeling. Nucleic Acids Res 2011, 39 (Web Server issue), W210-4.

44. Phillips, J. C.; Braun, R.; Wang, W.; Gumbart, J.; Tajkhorshid, E.; Villa, E.; Chipot, C.; Skeel, R. D.; Kale, L.; Schulten, K., Scalable molecular dynamics with NAMD. Journal of computational chemistry 2005, 26 (16), 1781-802.

45. Bonomi, M.; Branduardi, D.; Bussi, G.; Camilloni, C.; Provasi, D.; Raiteri, P.; Donadio, D.; Marinelli, F.; Pietrucci, F.; Broglia, R. A.; Parrinello, M., PLUMED: A portable plugin for free-energy calculations with molecular dynamics. Comput Phys Commun 2009, 180 (10), 19611972.

46. Vanommeslaeghe, K.; Hatcher, E.; Acharya, C.; Kundu, S.; Zhong, S.; Shim, J.; Darian, E.; Guvench, O.; Lopes, P.; Vorobyov, I.; MacKerell, A. D., CHARMM General Force Field: A Force Field for Drug-Like Molecules Compatible with the CHARMM All-Atom Additive Biological Force Fields. Journal of computational chemistry 2010, 31 (4), 671-690.

47. Frisch, M. J. T., G. W.; Schlegel, H. B.; Scuseria, G. E.; Robb, M. A.; Cheeseman, J. R.; Scalmani, G.; Barone, V.; Mennucci, B.; Petersson, G. A.; Nakatsuji, H.; Caricato, M.; Li, X.; Hratchian, H. P.; Izmaylov, A. F.; Bloino, J.; Zheng, G.; Sonnenberg, J. L.; Hada, M.; Ehara, M.; Toyota, K.; Fukuda, R.; Hasegawa, J.; Ishida, M.; Nakajima, T.; Honda, Y.; Kitao, O.; Nakai, H.; 
Vreven, T.; Montgomery, J. A., Jr.; Peralta, J. E.; Ogliaro, F.; Bearpark, M.; Heyd, J. J.; Brothers, E.; Kudin, K. N.; Staroverov, V. N.; Kobayashi, R.; Normand, J.; Raghavachari, K.; Rendell, A.; Burant, J. C.; Iyengar, S. S.; Tomasi, J.; Cossi, M.; Rega, N.; Millam, M. J.; Klene, M.; Knox, J. E.; Cross, J. B.; Bakken, V.; Adamo, C.; Jaramillo, J.; Gomperts, R.; Stratmann, R. E.; Yazyev, O.; Austin, A. J.; Cammi, R.; Pomelli, C.; Ochterski, J. W.; Martin, R. L.; Morokuma, K.; Zakrzewski, V. G.; Voth, G. A.; Salvador, P.; Dannenberg, J. J.; Dapprich, S.; Daniels, A. D.; Farkas, Ö.; Foresman, J. B.; Ortiz, J. V.; Cioslowski, J.; Fox, D. J. Gaussian 09, Revision D.01, Gaussian, Inc.: Wallingford CT, 2009.

48. Jorgensen, W. L.; Jenson, C., Temperature dependence of TIP3P, SPC, and TIP4P water from NPT Monte Carlo simulations: Seeking temperatures of maximum density. Journal of computational chemistry 1998, 19 (10), 1179-1186.

49. Nosé, S., A unified formulation of the constant temperature molecular-dynamics methods. J Chem Phys 1984, 1 (1), 511-519.

50. Hoover, W. G., Canonical dynamics: Equilibrium phase-space distributions. Phys Rev Lett 1985, 31 (3), 3.

51. Miyamoto, S.; Kollman, P. A., Settle: An analytical version of the SHAKE and RATTLE algorithm for rigid water models. Journal of computational chemistry 1992, 13 (8), 11. 